\title{
Quasinormal modes of the charged black hole in Gauss-Bonnet gravity
}

\author{
Roman Konoplya \\ Instituto de Fisica, Universidade de São Paulo, \\ C.P.66318, CEP 05315, São Paulo SP, Brazil \\ konoplya@fma.if.usp.br
}

\begin{abstract}
The d-dimensional string generated gravity models lead to EinsteinMaxwell equations with quadratic order correction term called the Gauss-Bonnet term. We calculate the quasinormal modes for the ddimensional charged black hole in the framework of this model. The quasinormal spectrum essentially depends upon the Gauss-Bonnet coupling parameter $\alpha$ which is related to the string scale, and is totally different from that for black holes derived from Einstein action. In particular, at large $\alpha$ the quasinormal modes are proportional to $\alpha$, and thus, have greater oscillation frequency and damping rate, while as $\alpha$ goes to zero the qusinormal modes approach their Schwarzschild values. In contrary to Einstein theory black hole behavior, the damping rate of the charged GB black hole as a function of charge does not contain a chracteristic maximum, but instead the monotonic falling down is observed. In addition, there have been obtained an asymptotic formula for large multipole numbers.
\end{abstract}




\section{Introduction}

Black holes in greater than four space-time dimensions have attracted a lot of interest within different theories. One of them is the supersymmetric string theory: its slope expansion yields corrections to the Einstein action. These corrections contain higher then the first powers of curvature. The quadratic term in curvature is the leading correcting term and can affect the graviton excitation spectrum near flat space. Thus one can consider the low-energy limit of string theories as an effective models of gravity in higher dimensions. The full low-energy theory must solve the problem of singularities in general relativity. The so called Gauss-Bonnet invariant is one of the most promising candidates for such quadratic curvature corrections to the Einstein action.

In addition, Lovelock came to this action when investigating the following question in classical theory of gravitation: what is the most general divergence free second rank symmetric tensor in D-dimensional space-time, constructed form the metric and its derivatives up to the second order?

The spherically symmetric solution describing neutral static black hole in Gauss-Bonnet gravity was obtained by Boulwar and Deser in [1]. The charged black hole solution was found by Wiltshire 2. Since that time, the properties of Gauss-Bonnet black holes were studied in a lot of papers 3]-15], with special interest to black holes in anti-de Sitter space-time as that having interpretation due to ADS/CFT correspondence.

As is known, one of the most important characteristic of a black hole, which play a fundamental role for both classical (see [16] for a review) and quantum [17] description is its quasinormal spectrum. The quasinormal modes are resonance oscillations which dominate at late times of the black hole response to an external perturbation. Recently a lot of papers (see for instance [18 - 35] and references therein) have been devoted to this subject motivated either by observational aspects or quantum aspects coming from Loop Quantum Gravity and ADS/CFT correspondence.

At the same time the higher dimensional black holes have been actively investigated recently within different extra dimensional scenarios (see 32 for a recent review). There, it is expected that the Planck scale is of order of $\mathrm{TeV}$, and thereby the quantum gravity may show itself at LHC where it is expected to observe mini-black holes. The Gauss-Bonnet black holes are being studied in this context as well (see [8] and references therein). In this connection, the quasinormal modes which are independent of the way of their excitation, and, thereby represent the "footprints" of a black hole, could give us the footprints" of the Gauss-Bonnet coupling, i.e. of low energy regime of quantum gravity. 
Therefore we are interested here, what will happen with quasinormal spectrum of the black hole if the Gauss-Bonnet correction term will be taken into consideration. To our surprise the first, and as far as we are aware the only, paper on this subject was written yet 15 years ago [33. There, some modes for few values of the slope parameter were evaluated when considering the scalar wave in the five and sixth dimensional neutral GaussBonnet black hole background. Here we are performing a thorough study of the Gauss-Bonnet black hole quasinormal modes for both neutral and charged black hole which include the whole range of the slope parameter $\alpha$, and different multipole numbers $l$ and different dimensionality of spacetime $D$. In particular, we investigate the asymptotic regime of large $\alpha$ and $l$. It is shown that the quasinormal behavior is totally different from that for the ordinary black hole corresponding to Einstein action. Thus, the quasinormal behavior is crucially depend upon the slope parameter $\alpha$ : the QN modes approach their Schwarzschild values when $\alpha$ goes to zero, and are proportional to $\alpha$ at large values of $\alpha$. Thus, influence of GaussBonnet (GB) coupling lead to increasing of the oscillation frequencies and to a greater decay rate in the regime of large GB coupling $\alpha$. For moderate values of $\alpha$, the the damping rate, as a function of $\alpha$, is decreasing reaches some minimum, and then increasing as $\sim \alpha$.

The dependence of the spectrum on the charge of the black hole is also different from that for Reissner-Nordstrom black hole: the imaginary part is just monotonically decreasing as the charge is increased, while for charged black holes derived from Einstein action such as Reissner-Nordstom black hole or its dilaton extension, one has the characteristic maximum of the imaginary part of $\omega$ somewhere near the 0.8 of its extremal value of charge.

The paper is organized as follows: in Sec.II we give some preliminary information on Gauss-Bonnet black hole perturbations. Section III deals with calculation of the quasinormal spectrum for Gauss-Bonnet black hole. Sec IV is devoted to summary of the result and some future perspectives of this work.

\section{Basic formulas}

The Lagrangian for the Einstein action and the Gauss-Bonnet action in the D-dimensional space-time model has the form:

$$
I=\frac{1}{16 \pi G_{D}} \int d^{D} x \sqrt{-g} R
$$




$$
+\alpha^{\prime} \int d^{D} x \sqrt{-g}\left(R_{a b c d} R^{a b c d}-4 R_{c d} R^{c d}+R^{2}\right)
$$

where $\alpha \prime$ is (positive) Gauss-Bonnet coupling constant which is related to the Regge slope parameter or string scale.

The black hole solution for this action is described by the metric:

$$
d s^{2}=-f(r) d t^{2}+f^{-1}(r) d r^{2}+r^{2} d \Omega_{D-2}^{2} .
$$

Here the asymptotically flat solution corresponds to the following form of the function $f[1]$ :

$$
f(r)=1+\frac{r^{2}}{2 \alpha}-\frac{r^{2}}{2 \alpha} \sqrt{1+\frac{8 \alpha \mu}{r^{D-1}}} .
$$

Here $\mu$ is the integration constant which is related to the black hole mass, and, $\alpha$ and $\alpha$ are connected by the relation:

$$
\alpha=16 \pi G_{D}(D-3)(D-4) \alpha \prime
$$

Note that in four space-time dimensions the Gauss-Bonnet term is the topological invariant 36]. The gravitational and thermodynamic properties of the above black holes were studied in a lot of papers recently [3], 4], [10, 11], 12, 14, 15], including the extension to the non-zero lambda term case. There are some range of parameter $\alpha$ and $\mu$ for which a black hole shows either thermodynamical or gravitational ( or both) instability [10], 14. Yet for $D>6$ and $D=5$ the Gauss-Bonnet black hole is stable against tensor type of gravitational perturbations 13, which, as was shown by Gibbons and Hartnoll [37] is, in a sense, the decisive type of gravitational perturbations which produce instability at least in the Einstein theory black holes. The charged Gauss-Bonnet black hole corresponds to the following form of the function $f$ [2]:

$$
f(r)=1+\frac{r^{2}}{2 \alpha}-\frac{r^{2}}{2 \alpha} \sqrt{1+\frac{8 \alpha \mu}{r^{D-1}}-\frac{4 \alpha Q^{2}}{2 \pi(D-2)(D-3) r^{2 D-4}}} .
$$

Here $Q$ is the charge of the black hole. In the latter case, there will be timelike singularity shielded by two horizons which tend to coincide as the charge $Q$ goes to its extremal values $Q_{\text {ext }}$. The extremal value of charge is determined by the relation:

$$
r_{e x t}^{2(D-3)}+\alpha \frac{D-5}{D-3} r_{e x t}^{2(D-4)}-\frac{Q_{e x t}^{2}}{2 \pi(D-2)(D-3)}=0
$$


where

$$
r_{e x t}^{D-3}=-\frac{1}{3}(D-5) \mu+\left(\frac{1}{4}(D-5)^{2} \mu^{2}+\frac{(D-4) Q_{e x t}^{2}}{2 \pi(D-2)(D-3)}\right)^{1 / 2} .
$$

To consider scalar perturbations in the background of the above GaussBonnet black hole we need to consider the test scalar field in the GB black hole background, i.e the consider the Klein-Gordon-Fock equartion:

$$
\square \Phi=\frac{1}{\sqrt{-g}}\left(g^{\mu \nu} \sqrt{-g} \Phi,{ }_{\mu}\right){ }_{\nu}=0 .
$$

After the change of the wave function $\Phi$, and going over to the tortoise coordinate $d r^{*}=d r / f(r)$, the last equation can be reduced to the Schrödinger wave-like form:

$$
\left(\frac{d^{2}}{d r^{* 2}}+\omega^{2}-V\left(r^{*}\right)\right) \Psi=0 .
$$

An effective potential has the form

$$
V(r)=f(r)\left(\frac{(D-2)(D-4)}{4 r^{2}} f(r)+\frac{D-2}{2 r} f^{\prime}(r)+\frac{l(l+D-3)}{r^{2}}\right)
$$

The tortoise coordinate $r^{*}$ is defined on the interval $(-\infty,+\infty)$ in such a way that the spatial infinity $r=+\infty$ corresponds to $r^{*}=\infty$, while the event horizon corresponds to $r^{*}=-\infty$. The above effective potential is positively defined and has the form of the potential barrier which approaches constant values at both spatial infinity and event horizon.

\section{Quasinormal modes of GB black hole}

Under the choice of the positive sign of the real part of $\omega(\omega=\operatorname{Re} \omega-\operatorname{Im} \omega)$, QNMs satisfy the following boundary conditions

$$
\Psi\left(r^{*}\right) \sim C_{ \pm} \exp \left( \pm i \omega r^{*}\right), \quad r \longrightarrow \pm \infty
$$

corresponding to purely in-going waves at the event horizon and purely outgoing waves at infinity.

To find the quasinormal modes of the black hole whose the effective potential has the form of potential barrier like that of the formula (9), one can use the WKB approach. The latter was first used for calculations of quasinormal modes in the first order by Schutz and Will [38, and extended 
by Iyer and Will to the third order beyond the eikonal approximation 39, and then extended to sixth WKB order in 40. The WKB approach for finding QN modes is used in a lot of papers, and in particular the WKB corrections up to 6 th order have been used recently in [43, 41, 42]. The WKB formula has the form [40]:

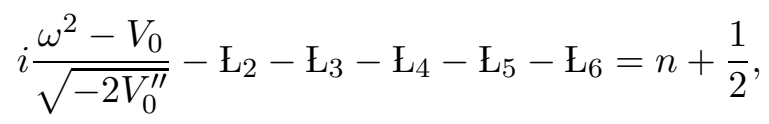

where $V_{0}$ is the height and $V_{0}^{\prime \prime}$ is the second derivative with respect to the tortoise coordinate of the potential at the maximum. $\mathrm{E}_{2}$ and $\mathrm{E}_{3}$ can be found in [39], $\mathrm{七}_{4}, \mathrm{七}_{5}$ and $\mathrm{E}_{6}$ are presented in [40; the corrections depend on the value of the potential and higher derivatives of it at the maximum. Below we shall discuss the obtained qusinormal modes classified according to the multipole number for charged and neutral GB black hole.

From here and on we shall take $\mu=1$ for any dimension $D$, thus, in different space-time dimensions the units are different.

\subsection{The neutral black hole, $\mathrm{l}=0$}

Within the WKB approach, we shall use here, $l=0$ modes represent an exclusive case, since the unmodified WKB method is a good approximation when $l>n$, being not so good for $l=n$ (and in fact being inapplicable when $l>n)$. As is known from comparison with numerical computations [39, the error at $l=0$ scalar modes can reach even 10 per cent for the Schwarzshild black hole case. So one should be careful when interpreting the WKB results for this case. The $l=0$ modes for $d=5,6,7,8$-dimensional black holes are represented in the following tables for different values of $\alpha$ : 


\begin{tabular}{|c|c|c||c|c|c|}
\hline$\alpha(D=5)$ & $\operatorname{Re} \omega$ & $-\operatorname{Im} \omega$ & $\alpha(D=7)$ & $\operatorname{Re} \omega$ & $-\operatorname{Im} \omega$ \\
\hline \hline $1 / 10$ & 0.389935 & 0.256159 & $1 / 10$ & 1.11738 & 0.546056 \\
\hline $1 / 5$ & 0.396034 & 0.250548 & $1 / 5$ & 1.13699 & 0.529543 \\
\hline $1 / 2$ & 0.429741 & 0.208293 & $1 / 2$ & 1.29469 & 0.395111 \\
\hline 5 & - & - & 5 & 1.39823 & 0.574472 \\
\hline 10 & - & - & 10 & 1.48053 & 0.385616 \\
\hline 20 & - & - & 20 & 2.00368 & 0.56458 \\
\hline \hline$\alpha(D=6)$ & $\operatorname{Re} \omega$ & $-\operatorname{Im} \omega$ & $\alpha(D=8)$ & $\operatorname{Re} \omega$ & $-\operatorname{Im} \omega$ \\
\hline \hline $1 / 10$ & 0.735854 & 0.402416 & $1 / 10$ & 1.51702 & 0.694245 \\
\hline $1 / 5$ & 0.748053 & 0.391049 & $1 / 5$ & 1.54463 & 0.673566 \\
\hline $1 / 2$ & 0.83530 & 0.304837 & $1 / 2$ & 1.7854 & 0.488086 \\
\hline 5 & 0.906661 & 0.144820 & 5 & 1.47941 & 0.868859 \\
\hline 10 & 1.513624 & 0.456935 & 10 & 1.800 & 0.410252 \\
\hline 20 & 2.961675 & 0.927128 & 20 & 2.15426 & 0.556954 \\
\hline
\end{tabular}

Table $1 \quad(L=0)$

We presented here a fundamental $(n=0)$ overtone which is dominating in a signal. Some discussions of higher overtone behavior will be given in the following subsections. Note that in five space-time diamnsions we have a black hole-type solution only for $\alpha<1.9$, that is why the corresponding boxes in the table are empty.

\subsection{The neutral black hole, $l=1,2$ and asymptotically high $l$}

The case of higher than zero multipoles is favorable for WKB methods. Here we observe good convergence of the $\mathrm{QN}$ values when increasing the $\mathrm{WKB}$ order. The $l=1,2$ fundamental $\mathrm{QN}$ modes are given in the tables 2 and 3 respectively. 


\begin{tabular}{|c|c|c||c|c|c|}
\hline$\alpha(D=5)$ & $\operatorname{Re} \omega$ & $-\operatorname{Im} \omega$ & $\alpha(D=7)$ & $\operatorname{Re} \omega$ & $-\operatorname{Im} \omega$ \\
\hline \hline $1 / 10$ & 0.720423 & 0.255506 & $1 / 10$ & 1.54573 & 0.577608 \\
\hline $1 / 5$ & 0.723177 & 0.252684 & $1 / 5$ & 1.53194 & 0.58701 \\
\hline $1 / 2$ & 0.739205 & 0.236885 & $1 / 2$ & 1.56277 & 0.554551 \\
\hline 5 & - & - & 5 & 2.01379 & 0.308316 \\
\hline 10 & - & - & 10 & 2.47824 & 0.423737 \\
\hline 20 & - & - & 20 & 3.39094 & 0.59452 \\
\hline \hline$\alpha(D=6)$ & $\operatorname{Re} \omega$ & $-\operatorname{Im} \omega$ & $\alpha(D=8)$ & $\operatorname{Re} \omega$ & $-\operatorname{Im} \omega$ \\
\hline \hline $1 / 10$ & 1.139007 & 0.415034 & $1 / 10$ & 1.93407 & 0.750046 \\
\hline $1 / 5$ & 1.136193 & 0.415706 & $1 / 5$ & 1.90391 & 0.773947 \\
\hline $1 / 2$ & 1.158635 & 0.391339 & $1 / 2$ & 1.94463 & 0.734591 \\
\hline 5 & 1.790103 & 0.258382 & 5 & 2.44511 & 0.455166 \\
\hline 10 & 3.260415 & 0.498426 & 10 & 2.6644 & 0.463966 \\
\hline 20 & 6.437139 & 0.991374 & 20 & 3.2276 & 0.581024 \\
\hline
\end{tabular}

Table $2 \quad(L=1)$

\begin{tabular}{|c|c|c||c|c|c|}
\hline$\alpha(D=5)$ & $\operatorname{Re} \omega$ & $-\operatorname{Im} \omega$ & $\alpha(D=7)$ & $\operatorname{Re} \omega$ & $-\operatorname{Im} \omega$ \\
\hline \hline $1 / 10$ & 1.07492 & 0.248021 & $1 / 10$ & 2.10314 & 0.525815 \\
\hline $1 / 5$ & 1.08169 & 0.243352 & $1 / 5$ & 2.10106 & 0.524304 \\
\hline $1 / 2$ & 1.10459 & 0.228154 & $1 / 2$ & 2.10473 & 0.51385 \\
\hline 5 & - & - & 5 & 2.66996 & 0.330935 \\
\hline 10 & - & - & 10 & 3.41504 & 0.433789 \\
\hline 20 & - & - & 20 & 4.69346 & 0.603341 \\
\hline \hline$\alpha(D=6)$ & $\operatorname{Re} \omega$ & $-\operatorname{Im} \omega$ & $\alpha(D=8)$ & $\operatorname{Re} \omega$ & $-\operatorname{Im} \omega$ \\
\hline \hline $1 / 10$ & 1.60284 & 0.39205 & $1 / 10$ & 2.58782 & 0.651764 \\
\hline $1 / 5$ & 1.607508 & 0.387098 & $1 / 5$ & 2.57296 & 0.658318 \\
\hline $1 / 2$ & 1.62621 & 0.370114 & $1 / 2$ & 2.54771 & 0.665021 \\
\hline 5 & 2.619279 & 0.269015 & 5 & 3.06761 & 0.428616 \\
\hline 10 & 4.81752 & 0.505708 & 10 & 3.49737 & 0.47698 \\
\hline 20 & 9.52384 & 1.00306 & 20 & 4.26009 & 0.589931 \\
\hline
\end{tabular}

Table $3 \quad(L=2)$

For large multipole number $l$ we can obtain the asymptotic formula using the first order WKB approach. For this to perform we first represent the 


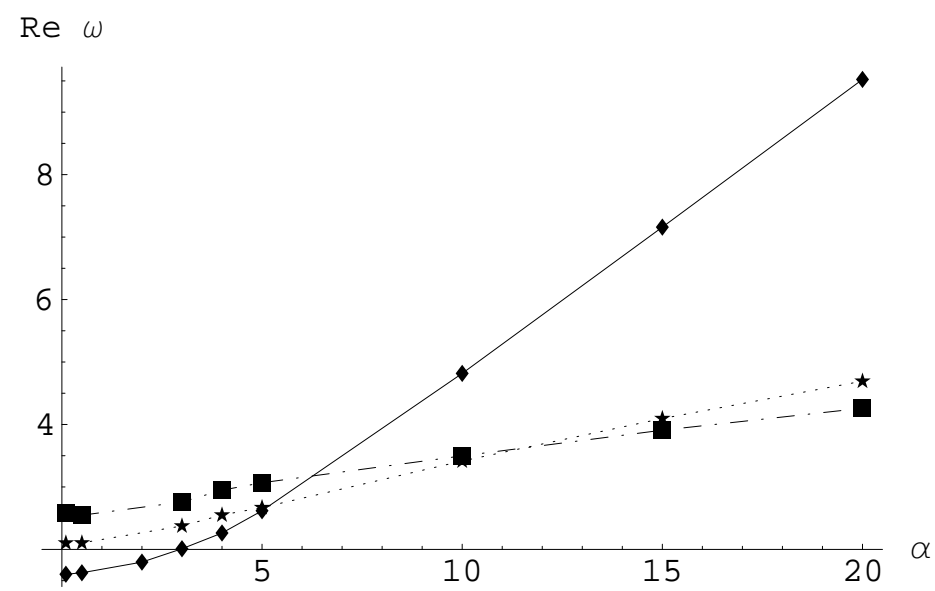

Figure 1: Real part of $\omega$ as a function of the Gauss-Bonnet coupling constant $\alpha$ for $l=2, n=0$ modes of $\operatorname{six}($ diamond), seven(star) and eight(box) dimensional black holes

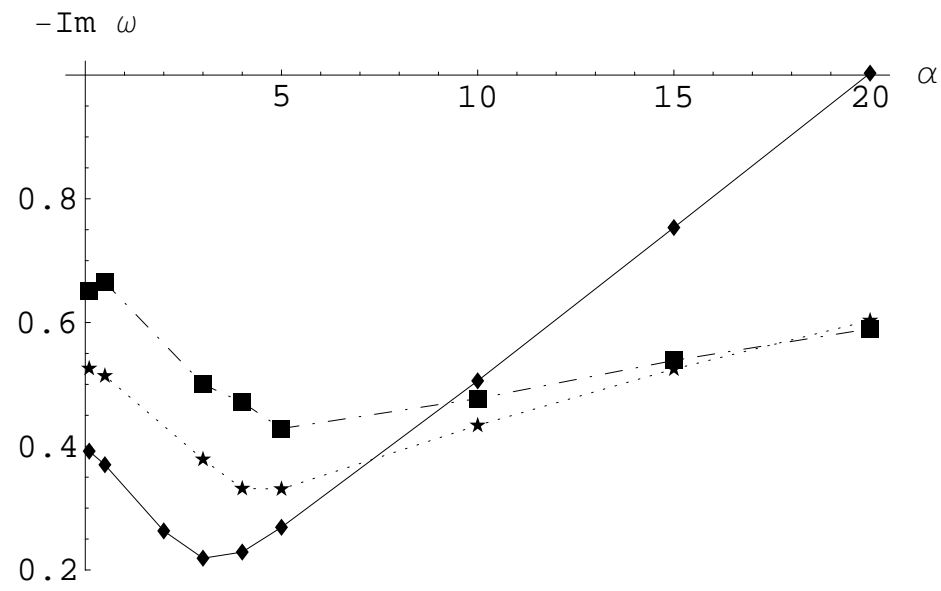

Figure 2: Imaginary part of $\omega$ as a function of the Gauss-Bonnet coupling constant $\alpha$ for $l=2, n=0$ modes of $\operatorname{six}($ diamond), seven(star) and eight(box) dimensional black holes 
value of $r$ at which the effective potential attains the maximum in the form:

$$
r_{\max }=r_{0}+r_{1}(1 / l)+r_{2}(1 / l)^{2}+\ldots
$$

Then we expand the effective potential and its second derivative in orders $1 / l$ and insert this exapnsded values into the first order WKB formula. As a result for the most slow damping modes we obtain the asymptotic formula:

$$
\omega_{R e}=\left(\frac{1}{r_{0}^{2}}-\frac{-1+\sqrt{1+8 r_{0}^{1-D} \alpha M}}{2 \alpha}\right) l+A, \quad \omega_{I m}=B
$$

where $A$ and $B$ are constants which does not depend on $l$ but depends only on black hole parameters and determined by cumbersome relations and therefore are not given here.

To show that as $\alpha$ goes to zero the QN modes of GB black hole goes to their Schwarzschild values, let us look at the fit for some sample mode $(D=5, l=2, n=0)$, built on five values of $\alpha, \alpha=1 / 100,1 / 200, \ldots 1 / 500$ :

$$
\begin{gathered}
\omega=1.0681-0.2528 i+ \\
(0.0422+0.0055 i) \alpha+(2.4021+3.8373 i) \alpha^{2}+O\left[\alpha^{3}\right] .
\end{gathered}
$$

We see that the fit is approching the corresponding Schwarzschild value $1.0681-0.2529 i$.

(Note that in 42] the tensor type gravitational modes are given which coincide with scalar field modes found in 40]).

This result can be easily explained by the fact that for small $\alpha$ one has an asymptotic

$$
f(r)=1-\frac{2 M}{r^{D-3}}+\frac{4 \alpha M}{r^{2 D-4}}+O\left[\alpha^{2}\right], \quad \alpha \rightarrow 0,
$$

and therefore as $\alpha$ goes to zero the metric approaches the Schwarzschild metric.

The dependence of the quasinormal modes on $\alpha$ can be seen from sample figures 1 and 2 and all the above data. Namely we see that when $\alpha$ is vanishing, both real and imaginary parts approach its higher dimensional Schwarzschild values, while when $\alpha$ is becoming large, the quasinormal frequencies are proportional to $\alpha$. Apparently it is possible to obtain some analytic formula for this regime of large $\alpha$. Note that one can observe the convergence of the results obtained with the WKB formula when increasing the WKB order for both large and very small $\alpha$. The worst convergence 
occurs for intermediate values of $\alpha$, yet this happens only for zero multipole modes when $l=n$. For $l>n$ the convergence is good for the whole range of parameters. Note that the WKB method in the Will-Schutz form uses the series which is convergent only asymptotically, therefore we are unable estimate the error strictly within used method. Yet, in principle, it can be modified to control the error [48. All previous expiriance in usage of the Will-Schutz approach, based on comparison with accurate numerical results, says that if we observe the convergence, then it is the convergence to some true QN value, and the greater the WKB order the better the accuracy. Certainly we do not have any garantee that we shall observe the convergence in any case we wish.

Thus from (12) we see that for the GB black hole, similar to the higher dimensional Schwarzschild black hole behavior, for asymptotically large multipole $l$, the real part is proportional to $l$ while the imaginary part approaches some constant. The higher overtones within WKB method used here can be obtained only with $l>n$. For instance, from the following data for sufficiently large multipole $(l=5)$ we can reproduced first several overtones:

$$
\begin{array}{ll}
4.73161-0.34419 i & n=0 \\
4.70975-1.02805 i & n=1 \\
4.64921-1.69859 i & n=2 \\
4.49639-2.35764 i & n=3 \\
4.13195-3.05609 i & n=4
\end{array}
$$

In the above data $\alpha=5$, and $D=7$. On this example data we see that the first few higher overtones have decreasing real part as the imaginary part increases. That feature is similar to the Schwarzschild black hole quasinormal behavior. To find sufficiently high overtones one should use the continued fraction technique.

\subsection{The charged black hole}

The dependence of the quasinormal spectrum on the charge of the GB black hole is quite different from the case of the Einstein black holes. In particular, from [44, 45], 35] we know that the imaginary part of the quasinormal mode for Reissner-Nordstrem or dilaton black holes is growing as the charge is increasing until some its maximum value, then, usually at the charge equaled approximately to $0.8 Q$ there is a sharp fall down. As can be seen from the Fig. 3, there is no such a maximum value of the damping rate, 


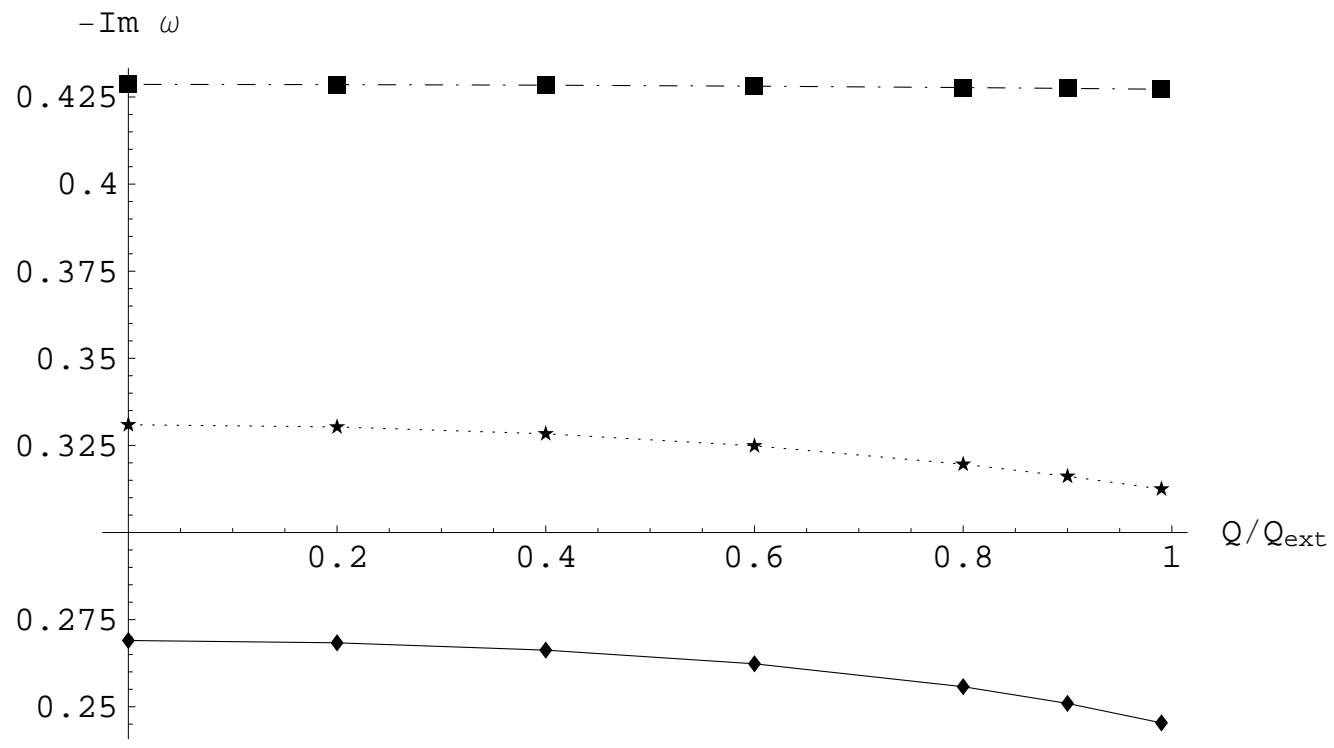

Figure 3: Imaginary part of $\omega$ for $\mathrm{D}=6$ (diamond), 7(star), 8 (box) dimensional black holes on example of $L=2, n=0, \alpha=5$.

but instead, the monotonic decreasing exists. On the Fig.4 one can see that the real oscillation frequency is just monotonically increases as the charge of the black hole is increased.

At high $l$ and non extremal value of the black hole charge, in a similar fashion to the case of the neutral black hole, we obtain the asymptotic formula:

$$
\omega_{R e}=\left(\frac{1}{r_{0}^{2}}-\frac{-1+\sqrt{1+8 r_{0}^{1-D} \alpha M-\frac{2 Q^{2} r_{0}^{4-2 D} \alpha}{6-5 D+D^{2}}}}{2 \alpha}\right)^{1 / 2} l+A^{\prime}, \quad \omega_{I m}=B^{\prime}
$$

where the constants $A^{\prime}$ and $B^{\prime}$ reduces to $A$ and $B$ when $Q=0$. The explicit form of these constants and of the coefficient $r_{0}, r_{1}$..etc. is available form the author upon request. 


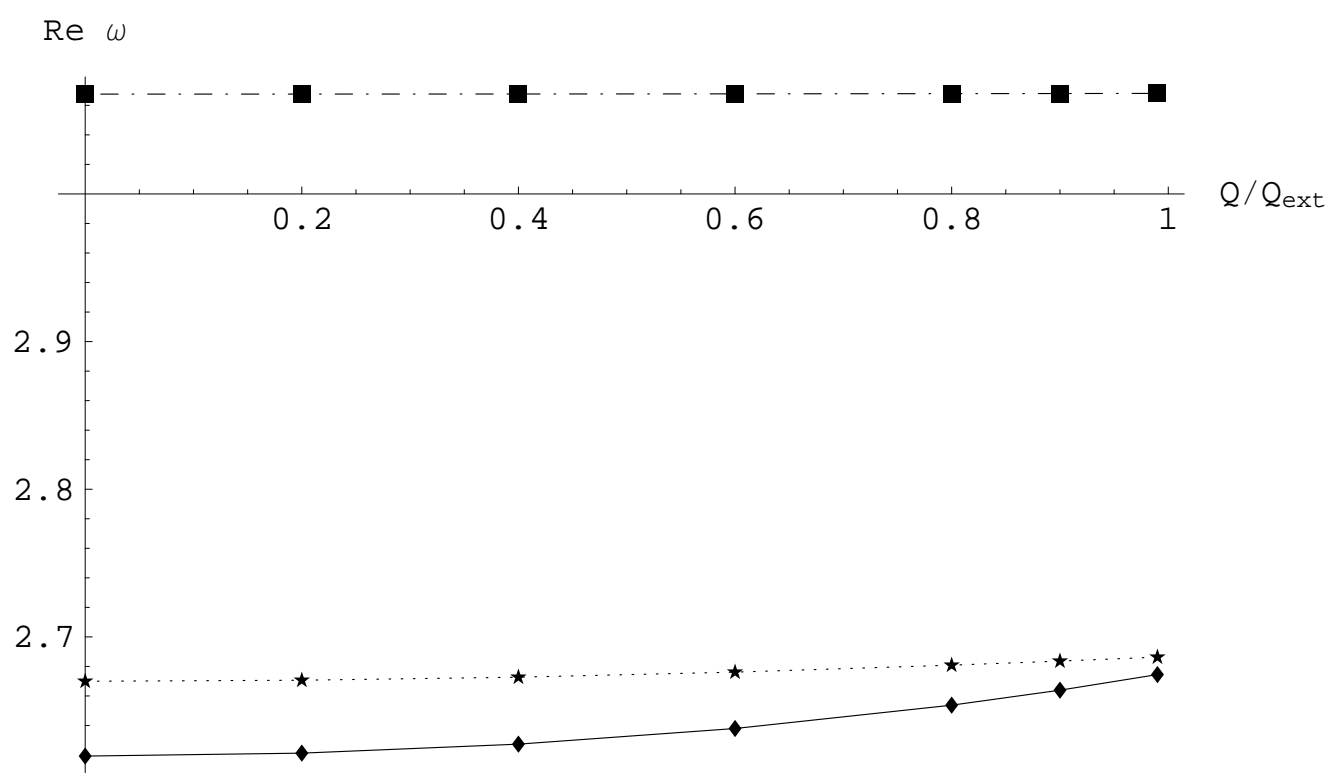

Figure 4: Real part of $\omega$ for $\mathrm{D}=6$ (diamond), 7(star), 8(box) dimensional black holes on example of $L=2, n=0, \alpha=5$ 


\section{Discussions}

We have investigated quasinormal modes of a black hole in Gauss-Bonnet gravity. It is shown that quasinormal behavior is totally different from that of black hole in Einstein gravity. In particular, the quasinoraml spectrum essetailly depends on the Gauss-Bonnet coupling parameter: at large $\alpha$ the QN modes are proportional to $\alpha$, while when $\alpha$ goes to zero, the QN modes approach their Schwarzschild values. Due to the Gauss-Bonnet coupling the quasinormal modes have greater oscillation frequency and greater damping rate at large $\alpha$, but at moderate $\alpha$, the damping rate as a function of $\alpha$, first, is decreasing until some minimum value and then begin to grow by the low $\sim \alpha$. As can be seen from comparison of the scalar filed potential considered here with that found in [14, for tensor-type gravitational perturbations, the scalar field potential and the tensor type gravitational potential coincide. The same situation takes place for higher dimensional black holes in Einstein gravity [46. Thus the quasinormal spectra are the same for adequate multipoles. If to disentangle equations for other types of gravitational perturbations of GB black hole is a solvable problem (see for instance references in [46], 47]), then the question of stability and gravitational QN radiation would be completed.

The problem that was beyond the present study is the finding of the asymptotically high overtones of the QN spectrum. After all, the quasinormal modes for the GB black hole in Anti-de Sitter space-time may have the $\mathrm{ADC} / \mathrm{CFT}$ holographic interpretation and are also interesting to study. We hope that further investigation will clarify these points.

\section{Acknowledgements}

The author would like to thank Ishware Neupanee for useful discussions. This work was supported by FAPESP (Brazil).

\section{References}

[1] D.G.Boulware and S.Deser, Phys. Rev. Lett. 55, 2656 (1985)

[2] D.Wiltshire, Phys.Rev. D38 2445 (1988).

[3] Rong-Gen Cai ei, Qi Guo, Phys.Rev.D69 104025, 2004 hep-th/0311020

[4] Rong-Gen Cai, Phys.Rev.D65 084014, 2002 hep-th/0109133 
[5] J. Crisostomo, R. Troncoso, J. Zanelli, Phys. Rev. D62 084013, 2000.

[6] Sachiko Ogushi, Misao Sasaki hep-th/0407083

[7] Tim Clunan, Simon F. Ross, Douglas J. Smith, Class.Quant.Grav.21 3447-3458, 2004 gr-qc/0402044

[8] A. Barrau, J. Grain, S.O. Alexeyev, Phys.Lett.B584 114, 2004 hep-ph/0311238

[9] Maximo Banados, Phys.Lett.B579 13-24, 2004 hep-th/0310160

[10] M. Cvetic, S. Nojiri, S. D. Odintsov, Nucl.Phys.B628 295, 2002 hep-th/0112045

[11] S. Nojiri, S. D. Odintsov, Phys.Lett.B523 165, 2001 hep-th/0110064

[12] S. Nojiri, S. D. Odintsov, S. Ogushi, Phys.Rev.D65 023521, 2002 hep-th/0108172

[13] Gustavo Dotti, Reinaldo J. Gleiser, gr-qc/0409005

[14] Ishwaree P. Neupane, Phys.Rev.D69 084011, 2004 hep-th/0302132

[15] Ishwaree P. Neupane, Phys.Rev.D67 061501, 2003 hep-th/0212092

[16] K.Kokkotas and B.Schmidt, "Quasi-normal modes of stars and black holes", Living. Reviews.Relativ . 2, 2 (1999)

[17] S.Hod Phys.Rev.Lett. 814293 (1998); (see also O. Dreyer, gr-qc/0211076).

[18] G.T.Horowitz and V.Hubeny, Phys. Rev. D62 024027 (2000)

[19] A. O. Starinets, Phys.Rev.D66 124013, 2002; A. Nunez, A. O. Starinets, Phys.Rev.D67 124013, 2003 e-Print Archive: hep-th/0302026

[20] L.Motl and A.Neitzke, Adv. Theor. Math. Phys. 7, 307 (2003);

[21] V.Cardoso and J.P.S.Lemos, Phys.Rev. D63 124015 (2001);

[22] R.A.Konoplya, Phys.Rev. D66 044009 (2002) hep-th/0205142; V. Cardoso, R. Konoplya and J. P. S. Lemos, Phys.Rev. D68 044024 (2003); R.A. Konoplya, Phys.Rev.D70:047503,2004 hep-th/0406100.

[23] C.Molina, D.Guingo, E.Abdalla, Phys. Rev. D69 104013 (2004); 
[24] H-B.Zhang, Z-J.Cao, X-F.Gong, W.Zhou, Class. Quant. Grav . 21 917 (2004);

[25] R.A.Konoplya and A.Zhidenko, JHEP (06) 037 (2004), hep-th/0402080 $\mathrm{v} 2]$.

[26] Sharmanthie Fernando, hep-th/0407062

[27] Bin Wang, Chi-Yong Lin, C. Molina, hep-th/0407024

[28] Mohammad R. Setare, Elias C. Vagenas, hep-th/0401187; T.Roy Choudhury, T. Padmanabhan , Phys. Rev. D 69064033 (2004) gr-qc/0311064; M. R. Setare, Class. Quant.Grav. 21 (2004) 1453 hep-th/0311221; M. R. Setare, Phys.Rev. D69 (2004) 044016 hep-th/0312061.

[29] George Siopsis, hep-th/0409262

[30] George Siopsis, hep-th/0408091

[31] Sharmanthie Fernando, Gen.Rel.Grav.36 71, 2004 hep-th/0306214

[32] Panagiota Kanti, hep-ph/0402168

[33] B.R. Iyer, S. Iyer, C.V. Vishveshwara, Class.Quant.Grav.6 1627, 1989

[34] R.A. Konoplya, Gen.Rel.Grav.34 329-335, 2002 gr-qc/0109096

[35] R.A.Konoplya, Phys.Rev. D66 084007 (2002), gr-qc/0207028.

[36] B. Zwiebach, Phys. Lett. B. 156, 315 (1985)

[37] Gary Gibbons, Sean A. Hartnoll, Phys.Rev.D66 064024, 2002 hep-th/0206202

[38] B.F.Schutz and C.M.Will, Astrophys. J. 291 L33 (1985)

[39] S. Iyer and C. M. Will, Phys.Rev. D35 3621 (1987)

[40] R.A.Konoplya, Phys.Rev. D68 024018 (2003)

[41] A.Zhidenko, Class.Quant.Grav. 21273 (2004)

[42] R.A. Konoplya, Phys.Rev.D68:124017,2003 hep-th/0309030

[43] Emanuele Berti, Vitor Cardoso, Jose P.S. Lemos, gr-qc/0408099 
[44] K.D. Kokkotas, B.F. Schutz, Phys.Rev.D37 3378-3387, 1988

[45] R.A. Konoplya, Phys.Lett.B550 117, 2002 gr-qc/0210105

[46] Hideo Kodama, Akihiro Ishibashi, gr-qc/0312012

[47] R.A. Konoplya, Phys.Lett.A268 37, 2000 gr-qc/9912101

[48] D.V. Gal'tsov and A.A. Matiukhin, Class. Quantum Grav. 92039 (1992) 